\title{
Retrospective Analysis of Clinical Characteristics and Neonatal Outcomes of Pregnant Women with SARS-COV-2 Infection*
}

\author{
Yu $\mathrm{CHEN}^{1 \dagger}$, Xiang-li $\mathrm{PANG}^{2 \dagger}$, Wen-ping $\mathrm{DING}^{3 \dagger}$, Xiang-chi $\mathrm{PENG}^{1}$, Jing $\mathrm{YANG}^{2 \#}$, Yan $\mathrm{ZHOU}^{4 \#}$ \\ ${ }^{1}$ Reproductive Medicine Center, Wuhan Children's Hospital (Wuhan Maternal and Child Healthcare Hospital), Tongji Medical \\ College, Huazhong University of Science and Technology, Wuhan 430015, China \\ ${ }^{2}$ Reproductive Medical Center, Renmin Hospital of Wuhan University, Wuhan 430060, China \\ ${ }^{3}$ Department of Diagnostic Ultrasound, Wuhan Children's Hospital (Wuhan Maternal and Child Healthcare Hospital), Tongji \\ Medical College, Huazhong University of Science and Technology, Wuhan 430015, China \\ ${ }^{4}$ Department of Obstetrics and Gynecology, Wuhan Children's Hospital (Wuhan Maternal and Child Healthcare Hospital), \\ Tongji Medical College, Huazhong University of Science and Technology, Wuhan 430015, China
}

(C) Huazhong University of Science and Technology 2021

\begin{abstract}
Summary: This retrospective study aimed to investigate the clinical characteristics and neonatal outcomes of pregnant women with SARS-COV-2 in Wuhan Children's Hospital and further suggested a possible management strategy for infected pregnant women under epidemic situation. In this study, 8 pregnant women with SARS-COV-2 who were admitted into Wuhan Children's Hospital, China from February 1, 2020 to March 30, 2020 and the clinical features, laboratory data, maternal and neonatal outcomes were analyzed. The mean age of the women at the time of admission was 30.6 years. The mean gestational age of the women was 37 weeks +4 days, and one woman presented with dichorionic diamniotic (DCDA) twin pregnancy. Except for one woman who was febrile, others had no typical clinical symptoms. For all pregnant women, the count of white blood cells and lymphocytes appeared normal, but 6 had a lower percentage of lymphocytes. $\mathrm{C}$-reactive protein (CRP) levels were normal for all the women. One neonate was tested positive for the coronavirus IgG and IgM antibodies. The clinical symptoms of the pregnant women with SARS-COV-2 were mild, and the laboratory data showed similar characteristics to those of non-infected pregnant women. Since one neonate was tested positive for coronavirus, there is a possibility of vertical transmission of SARS-CoV-2. Prompt and efficient screening, triage, and isolation of pregnant women are effective management strategies to reduce nosocomial infection during the SARS-COV-2 epidemic.
\end{abstract}

Key words: SARS-COV-2; vertical transmission; pregnant women; neonates; clinical features; maternal and child outcomes

At the end of 2019, a novel coronavirus severe acute respiratory syndrome coronavirus 2 (SARSCoV-2) first broke out. This virus belongs to the genus $\beta$, and primarily spreads via the respiratory droplets and through close contact. This lethal virus has quickly swept the globe due to its strong infectivity. By April 26, 2020, 2774135 people were positive for SARSCoV-2 and 190871 people died in at least 210 countries of the world ${ }^{[1]}$. Different populations have varying susceptibilities to the SARS-CoV-2. Some researchers

Yu CHEN, E-mail: 64812727@qq.com; Xiang-li PANG, E-mail: 489626237@qq.com; Wen-ping DING, E-mail: dwpkitty@163.com

†These authors contributed equally to this work.

\#Corresponding authors, Jing YANG, E-mail: dryangqing@, hotmail.com; Yan ZHOU, E-mail: zhouyan030118@126.com ${ }^{*}$ This project was supported by Wuhan Health Commission Fund, China (No. WX14A08). believe that male patients are more susceptible to SARS-COV-2 associated complications than female patients and this variation increases with age ${ }^{[2]}$. As per studies, the SARS-CoV-2 infection in pregnant women is mostly mild, and only a few cases of severe illness have been reported. The prognosis of pregnant women and newborns has appeared good ${ }^{[3,4]}$. Because obtaining the clinical information of pregnant women is difficult and the causative agent of SARS-COV-2 is completely novel, scientists are still exploring the possibilities of vertical transmission of SARS-CoV-2 from infected mother to her child. The average length of human gestation is 40 weeks; however, there are few reports on the prognosis of preterm delivery and multiple pregnancies with SARS-COV-2. The postadmission management of pregnant women with SARS-COV-2 is extremely crucial. In this study, we collected the clinical and laboratory data of 8 pregnant 
women and their newborns during the SARS-COV-2 epidemic in Wuhan, and described the management of pregnant women in hospitals during the epidemic so that the nosocomial infections are avoided as much as possible.

\section{MATERIALS AND METHODS}

\subsection{Study Participants and Diagnostic Criteria}

In this single-center, retrospective study, we collected the data of 8 pregnant women and their newborns who were hospitalized in Wuhan Children's Hospital from February 1, 2020 to March 30, 2020. For diagnosis, we followed the seventh edition of the new diagnosis and treatment scheme for SARSCOV-2 issued by the National Health Commission of the People's Republic of China ${ }^{[5]}$. All the patients were from the central area of the Wuhan city, Hubei province, China. After delivery, all the newborns were transferred to the SARS-COV-2 treatment area of the hospital. This study was approved by the Ethics Committee of Wuhan Children's Hospital and written informed consent was obtained from the patients.

\subsection{Data Collection}

We collected the relevant data about the pregnant women and neonates from the hospital medical records. The patients who were transferred to the SARS-COV-2 designated hospital after diagnosis were followed up through telephone to acquire the prognosis information and the clinical characteristics. Similarly, the data of neonates were collected from the medical records and telephone follow-ups. The laboratory tests (SARSCoV-2, chest CT, 3D reconstruction imaging etc.) of the pregnant women and newborns were carried out in the hospital. To ensure the accuracy of the data, all the data were rechecked by two investigators.

\section{RESULTS}

The age range of patients was 24-39 years, and the mean age was 30.6 years. The mean gestational age was 37 weeks +4 days (range 29 weeks +6 days -40 weeks). One of the patients of 29 weeks +6 days was admitted due to the premature rupture of membranes. All the patients were locals living in the Wuhan city, Hubei province, China. The family members of 3 pregnant women $(37.5 \%)$ were found to be positive for SARS$\mathrm{CoV}-2$. One patient had severe preeclampsia, one had gestational diabetes, one had oligohydramnios and one had a twin pregnancy. In total, 6 patients gave birth via a cesarean section (75\%) and 2 had vaginal delivery. Only one patient was febrile with body temperature of $37.7^{\circ} \mathrm{C}$. The rest of the patients had no fever or other respiratory symptoms. The chest CT of 3 patients showed typical ground-glass opacities (table 1).

The white blood cell counts of all pregnant women

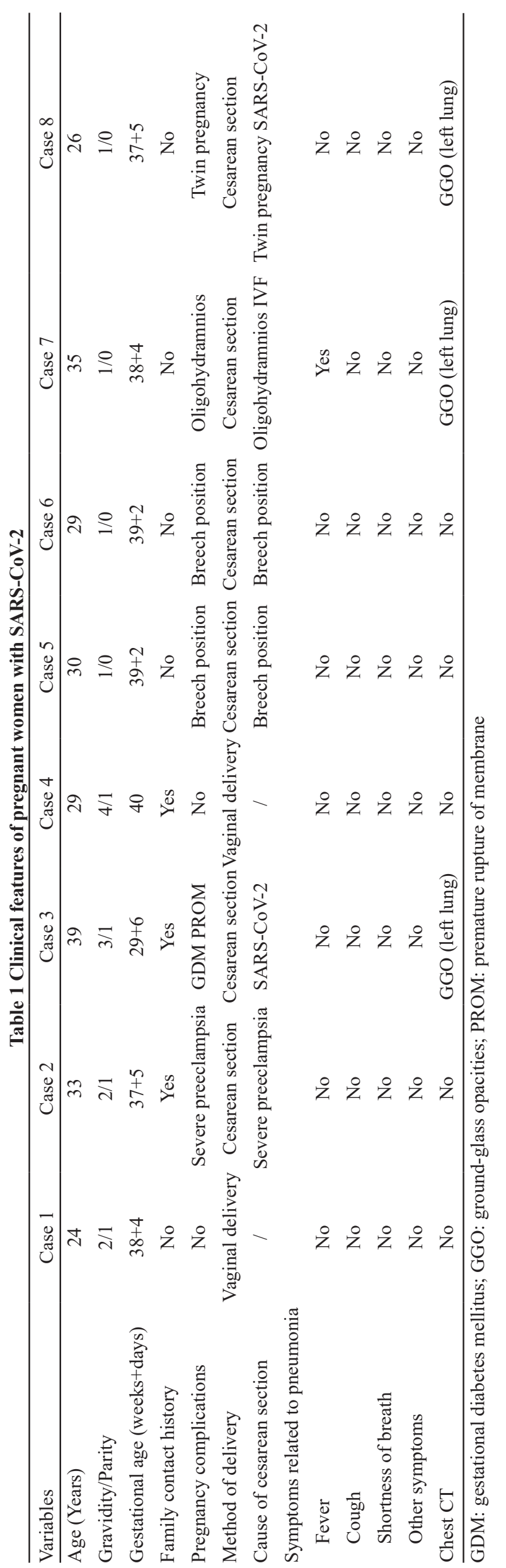


were normal or slightly elevated. The neutrophil count and its percentage was also normal or increased; lymphocyte count was normal, and the lymphocyte percentage was normal or low with no significant difference between the infected and non-infected pregnant women. C-reactive protein (CRP) was in normal range for all pregnant women. Five women were not tested for SARS-CoV-2 with the antibody test kits, but the results of their nucleic acid tests were positive. Three women were negative for SARS-CoV-2 in the nucleic acid tests, but their antibody tests for both IgG and IgM were positive (table 2).

Eight pregnant women gave birth to 9 neonates including 2 dichorionic diamniotic (DCDA) twins (37 weeks +5 days gestational age). Four mothers were hospitalized due to emergency, so they were not tested for SARS-COV-2 before delivery. The neonates had direct skin to skin contact with their mothers, and the mean rooming-in time was $24.7 \mathrm{~h}$ (range, 10-40 h). Four neonates were immediately transferred to the neonatology department after their mothers were tested positive for SARS-COV-2. The rest 5 neonates were immediately isolated and transferred to the neonatology department after birth. Six neonates were found to have hyperbilirubinemia, 3 had myocardial damage, one had necrotizing enterocolitis, and one premature infant had respiratory failure and bacterial sepsis (table $3)$. White blood cell counts for all the neonates were normal; 8 neonates had higher neutrophil count, and neutrophil percentage of all 9 neonates was higher. The lymphocyte count and its percentage were normal in all the newborns and the procalcitonin (PCT) was higher than normal. All the neonates were tested negative for coronavirus in nucleic acid tests; however, one neonate (gestational age 29 weeks +6 days) was positive for the $\mathrm{IgG}$ and IgM coronavirus antibodies, and 2 neonates were negative for IgG but positive for IgM (in table 4).

\section{DISCUSSION}

The clinical manifestations of SARS-COV-2 including asymptomatic infection, and mild respiratory illnesses such as influenza are non-specific, but severe SARS-COV-2 patients may have pneumonia or respiratory failure ${ }^{[6]}$. At present, SARS-COV-2 has turned into a pandemic. Many researchers believe that gender, age, and complications are associated with susceptibility ${ }^{[2,7,8]}$.

Pregnant women are one of the most vulnerable populations during an epidemic because of significant changes in the hormone levels and the immune system during pregnancy: the expression of SARS-CoV-2 receptor, ACE-2, increases during pregnancy ${ }^{[9]}$. Although pregnant women have a low risk of developing severe complications, some women still develop a severe illness ${ }^{[10]}$. Moreover, the possibility of vertical transmission from mother-to-child and an increase in risk of neonatal infection in case of vaginal

Table 2 Laboratory characteristics of pregnant women with SARS-CoV-2

\begin{tabular}{lccccccccc}
\hline Laboratory results & Reference value & Case 1 & Case 2 & Case 3 & Case 4 & Case 5 & Case 6 & Case 7 & Case 8 \\
\hline WBC count & $(4-15) \times 10^{9} / \mathrm{L}$ & 16.15 & 7.96 & 8.03 & 8.83 & 8.29 & 11.06 & 7.76 & 10.92 \\
Neutrophil count & $(2-7) \times 10^{9} / \mathrm{L}$ & 12.45 & 6.34 & 6.37 & 5.11 & 6.56 & 8.18 & 6.51 & 7.40 \\
Percentage of neutrophil & $50 \%-70 \%$ & 77.2 & 79.6 & 79.4 & 57.8 & 79.2 & 73.9 & 84 & 67.7 \\
Lymphocytes count & $(0.8-4) \times 10^{9} / \mathrm{L}$ & 2.68 & 1.13 & 1.24 & 2.70 & 1.26 & 2.12 & 1.47 & 2.35 \\
Percentage of lymphocyte & $20 \%-40 \%$ & 16.6 & 14.2 & 15.4 & 30.6 & 15.2 & 19.2 & 18.9 & 21.5 \\
CRP & $0-8$ & 7.20 & 5.6 & 0.7 & 1.23 & 0.6 & 5.0 & 6.87 & 5.27 \\
ALT & $9-52 / \mathrm{L}$ & 21 & 16 & 32 & 26 & 21 & 12 & 15 & 6 \\
AST & $15-46 / \mathrm{L}$ & 25 & 21 & 17 & 19 & 33 & 16 & 26 & 10 \\
SARS-CoV-2 IgG & $-/+$ & NA & + & + & + & + & NA & NA & NA \\
SARS-CoV-2 IgM & $-/+$ & NA & + & + & + & + & NA & NA & NA \\
SARS-CoV-2 nucleic acid & $-/+$ & + & - & - & - & + & + & + & + \\
\hline
\end{tabular}

CRP: C-reactive protein; ALT: alanine aminotransferase; AST: aspartate transaminase; NA: not available; WBC: white blood cells; CRP: C-reactive protein

Table 3 Clinical features of neonates

\begin{tabular}{|c|c|c|c|c|c|c|c|c|c|}
\hline Items & Case 1 & Case2 & Case 3 & Case4 & Case 5 & Case 6 & Case7 & $\begin{array}{l}\text { Case } 8 \\
\text { (twin 1) }\end{array}$ & $\begin{array}{l}\text { Case } 9 \\
\text { (twin 2) }\end{array}$ \\
\hline $\begin{array}{l}\text { Skin to skin contact between } \\
\text { mother and infant }\end{array}$ & Yes & Yes & No & Yes & No & Yes & No & No & No \\
\hline Delayed cord clamping & Yes & Yes & No & Yes & No & Yes & No & No & No \\
\hline Time of rooming-in (h) & 40 & 10 & 0 & 26 & 0 & 23 & 0 & 0 & 0 \\
\hline $\begin{array}{l}\text { Transfer to neonatology } \\
\text { department }\end{array}$ & Yes & Yes & Yes & Yes & Yes & Yes & Yes & Yes & Yes \\
\hline Related complications & $\begin{array}{l}\text { Myocardial } \\
\text { damage }\end{array}$ & NHB & $\begin{array}{l}\text { Severe asphyxia, } \\
\text { NHB, respiratory } \\
\text { failure, septicopyemia }\end{array}$ & / & $\begin{array}{l}\mathrm{NHB}, \\
\text { myocardial } \\
\text { damage }\end{array}$ & $\begin{array}{l}\mathrm{NHB}, \\
\text { myocardial } \\
\text { damage }\end{array}$ & / & $\begin{array}{l}\text { NEC; } \\
\text { NHB }\end{array}$ & NHB \\
\hline
\end{tabular}


Table 4 Laboratory characteristics of neonates

\begin{tabular}{|c|c|c|c|c|c|c|c|c|c|c|}
\hline Laboratory results & Reference value & Case 1 & Case 2 & Case 3 & Case 4 & Case 5 & Case 6 & Case 7 & Case 8 & Case 9 \\
\hline WBC count & $(5-30) \times 10^{9} / \mathrm{L}$ & 16.58 & 13.71 & 18.74 & 15.60 & 19.36 & 20.97 & 15.7 & 11.61 & 18.02 \\
\hline Neutrophil count & $(3.9-9.4) \times 10^{9} / \mathrm{L}$ & 10.7 & 9.82 & 12.57 & 9.8 & 13.45 & 14.46 & 10.52 & 9.42 & 14.59 \\
\hline Percentage of neutrophils & $31 \%-52 \%$ & 64.6 & 71.7 & 67.1 & 62.8 & 69.5 & 69 & 67 & 81.1 & 81.1 \\
\hline Lymphocytes count & $(2-17) \times 10^{9} / \mathrm{L}$ & 3.77 & 2.41 & 4.10 & 3.90 & 3.61 & 4.58 & 3.69 & 1.46 & 2.24 \\
\hline Percentage of lymphocyte & $31 \%-48 \%$ & 22.7 & 17.6 & 21.9 & 25 & 18.6 & 21.8 & 23.5 & 12.6 & 12.4 \\
\hline CRP & $0-3 \mathrm{mg} / \mathrm{L}$ & 3.14 & 1.21 & 0.9 & 1.5 & 0.45 & 0.5 & 0.66 & 0.55 & 0.33 \\
\hline PCT & $\leq 0.05 \mathrm{ng} / \mathrm{mL}$ & 1.29 & 6.9 & 0.16 & 0.20 & 0.88 & 2.62 & 0.57 & 0.29 & 0.43 \\
\hline ALT & $9-52 \mathrm{U} / \mathrm{L}$ & 22 & 22 & 7 & 30 & 23 & 32 & 42 & 19 & 21 \\
\hline AST & $15-46 \mathrm{U} / \mathrm{L}$ & 42 & 54 & 32 & 39 & 92 & 156 & 30 & 37 & 47 \\
\hline TBIL & $51-171 \mathrm{nmol} / 1$ & 129 & 123 & 89.2 & 101.5 & 75.1 & 98.1 & 62.5 & 52.6 & 55.1 \\
\hline DBIL & $0-10 \mathrm{nmol} / 1$ & 0 & 9.6 & 4.1 & 3.0 & 0 & 0 & 0 & 0 & 0 \\
\hline CK & $20-250$ & 177 & 381 & 109 & 121 & 134 & 358 & 186 & 421 & 357 \\
\hline LDH & $225-600$ & 314 & 326 & 471 & 340 & 334 & 753 & 322 & 352 & 502 \\
\hline SARS-CoV-2 IgG & $+1-$ & - & + & + & - & + & - & - & - & - \\
\hline SARS-CoV-2 IgM & $+1-$ & - & - & + & - & - & - & - & - & - \\
\hline SARS-CoV-2 nucleic acid & $+1-$ & - & - & - & - & - & - & - & - & - \\
\hline
\end{tabular}

CRP: C-reactive protein; PCT: procalcitonin; ALT: alanine aminotransferase; AST: aspartate transaminase; TBIL: total bilirubin; DBIL: direct bilirubin; CK: creatine kinase; LDH: lactate dehydrogenase

delivery is a matter of debate ${ }^{[11]}$.

In our study, all the pregnant women with SARSCOV-2 were either asymptomatic or showed mild symptoms. The white blood cell counts, neutrophil counts and percentage, lymphocyte counts and percentage and CRP observed in pregnant SARSCOV-2 women in our study were similar to those of non-pregnant asymptomatic and mild symptomatic women observed by Xu et al ${ }^{[12]}$.

Many researchers are against the possibility of vertical transmission of SARS-CoV-2 ${ }^{[13,14]}$, while others believe that the mother-to-child transmission is still possible ${ }^{[11,15]}$. In our study, twin neonates were not found to be infected with SARS-CoV-2, but 1 neonate was confirmed to have the infection: neonatal and maternal coronavirus IgG and IgM were positive. This infected baby (gestational age: 29 weeks +6 days), delivered by the cesarean section, was immediately isolated from the mother after birth. This proves that the risk of vertical transmission in SARS-COV-2 could not be ruled out and the possibility of transmission of SARS-CoV-2 from the mother to her child still exists.

Ferrazzi et al reported that the newborns delivered with a vaginal delivery of infected mothers have a lower chance of being infected than those delivered via a cesarean section ${ }^{[16]}$. Scorzolini et al believed that the cesarean section reduced the risk of neonatal infection $^{[17]}$. In our study, one neonate out of 6 born via a cesarean section was positive for both $\operatorname{IgG}$ and $\mathrm{IgM}$, and 2 neonates born by vaginal delivery were not infected. These 2 neonates had early contact with their mothers with rooming-in time of $40 \mathrm{~h}$ and $26 \mathrm{~h}$ respectively. This confirms that the vaginal delivery did not increase the risk of infection.

A study by Zhang et al concluded that neonates of infected mothers generally have a good prognosis with no obvious symptoms of infection ${ }^{[18]}$. In our study, 9 neonates were followed up and good prognosis was confirmed. The delivery of one infant was premature at 29 weeks +6 days of gestation, and 6 neonates had different kinds of complications. However, it is unknown if these complications were induced by the coronavirus or the neonates had some other infection. In the study, we also found 3 neonates with myocardial damage after birth, including 1 case (No. 5) negative for nucleic acid, $\operatorname{IgM}(-)$ but $\operatorname{IgG}(+)$, however, 2 cases (No. 1 and No. 6) negative for nucleic acid and antibody tests. Here we inferred that there was no correlation between myocardial damage and SARS$\mathrm{CoV}-2$ infection. Due to the limited number of cases, whether the specific myocardial damage is caused by SARS-CoV-2 infection needs to be further confirmed by the future studies.

SARS-COV-2, a global public health problem, is full of challenges for obstetricians. They have to be extra cautious and take everything into consideration while dealing with pregnant women with SARSCOV-2. Efficient triage and reasonable treatment process play a crucial role in taking care of the patients, avoiding nosocomial infection, and preventing maternal deaths ${ }^{[19-21]}$.

During the epidemic, social education system needs to be strengthened and measures should be taken to create awareness about maternal health. Besides, the importance of staying at home for pregnant women should be emphasized, and social distancing practices should be implemented. When pregnant women with the gestational age of over 38 weeks visit the hospital for antepartum examination, SARS-COV-2 testing by nucleic acid tests and chest-CT should also be performed. The pregnant women should be screened to avoid missing diagnosis and nosocomial 
infections when they are admitted to the hospital due to emergency labor. Pregnant women with positive SARS-COV-2 tests should be promptly transferred to the designated hospital. Moreover, hospitals need to set up specialized isolation wards, isolation delivery rooms, laminar negative pressure operation room, and special passageway for movement of the pregnant women with SARS-COV-2. The pregnant women who are not preliminary screened should be admitted to the isolation ward and timely tested for SARS-COV-2 by nucleic acid tests, antibody tests, and chest-CT scans. The obstetric situation of the patients should be dealt with first. If the SARS-COV-2 test results turn out to be positive, the obstetric situation should be stabilized before transferring the patients to the designated hospitals. The pregnant women should be given some specific treatment such as strengthening support therapy; ensuring sufficient caloric intake for patients; monitoring their electrolyte balance to maintain internal environment stability; closely monitoring vital signs and oxygen saturation; oral administration of Abidol tablets $(200 \mathrm{mg}$, three times a day for adults, no longer than 10 days). The neonates of infected women should be admitted to the neonatology department for further treatment. When the condition of neonates becomes stable, they should be transferred to the designated hospital with special care. The mothers should be followed up twice a week for initial few weeks and once a month later to check their prognoses, and the health status of each neonate was monitored for 14 days when it came to good prognosis ${ }^{[22]}$.

Our study is limited in terms of number of participants and the lack of information on tissues and body fluid samples such as cord blood, placental tissue, milk, vaginal secretions, etc. of infected pregnant women. In the future, we are planning a multi-center study to understand the pathogenesis of SARS-COV-2 and gather more evidence of vertical transmission of SARS-CoV-2.

\section{Conflict of Interest Statement}

We declare that we do not have any commercial or associative interest that represents a conflict of interest in connection with the work submitted.

\section{REFERENCES}

1 WHO. Coronavirus disease 2019 (SARS-COV-2) Situation Report. Available at https://www.who. int/emergencies/diseases/novel-coronavirus-2019. Accessed at 2020.

2 Meng Y, Wu P, Lu W, et al. Sex-specific clinical characteristics and prognosis of coronavirus disease-19 infection in Wuhan, China: A retrospective study of 168 severe patients. PLoS Pathog, 2020,16(4):e1008520

3 Yu N, Li W, Kang Q, et al. Clinical features and obstetric and neonatal outcomes of pregnant patients with COVID-19 in Wuhan, China: a retrospective, singlecentre, descriptive study. Lancet Infect Dis, 2020,20(5):
$559-564$

4 Iqbal $\mathrm{SN}$, Overcash $\mathrm{R}$, Mokhtari $\mathrm{N}$, et al. An Uncomplicated Delivery in a Patient with Covid-19 in the United States. N Engl J Med, 2020,382(16):e34

5 National Health Commission \& State Administration of Traditional Chinese Medicine of the People's Republic of China. Diagnosis and Treatment Protocol for Novel Coronavirus Pneumonia (Trial Version 7). (http://www. nhc.gov.cn/). 2020.

6 Wang D, Hu B, Hu C, et al. Clinical Characteristics of 138 Hospitalized Patients With 2019 Novel Coronavirus-Infected Pneumonia in Wuhan, China. JAMA, 2020,323(11):1061-1069

7 Niu S, Tian S, Lou J, et al. Clinical characteristics of older patients infected with COVID-19: A descriptive study. Arch Gerontol Geriatr, 2020,89:104058

8 Team CC-R. Severe Outcomes Among Patients with Coronavirus Disease 2019 (COVID-19) - United States, February 12-March 16, 2020. MMWR Morb Mortal Wkly Rep, 2020,69(12):343-346

9 Zhao X, Jiang Y, Zhao Y, et al. Analysis of the susceptibility to COVID-19 in pregnancy and recommendations on potential drug screening. Eur J Clin Microbiol Infect Dis, 2020,39(7):1209-1220

10 Pacheco LD, Saad AF, Saade G. Early Acute Respiratory Support for Pregnant Patients with Coronavirus Disease 2019 (COVID-19) Infection. Obstet Gynecol, 2020, 136(1):42-45

11 Dong L, Tian J, He S, et al. Possible Vertical Transmission of SARS-CoV-2 from an Infected Mother to Her Newborn. JAMA, 2020,323(18):1846-1848

12 Xu QC, Shen J, Pan LL, et al. Coronavirus disease 2019 in pregnancy. Int J Infect Dis, 2020,95:376-383

13 Lu D, Sang L, Du S, et al. Asymptomatic COVID-19 infection in late pregnancy indicated no vertical transmission. J Med Virol, 2020,92(9):1660-1664

14 Yan J, Guo J, Fan C, et al. Coronavirus disease 2019 in pregnant women: a report based on 116 cases. Am J Obstet Gynecol, 2020,223(1):111 e111-111 e114

15 Alzamora MC, Paredes T, Caceres D, et al. Severe COVID-19 during Pregnancy and Possible Vertical Transmission. Am J Perinatol, 2020,37(8):861-865

16 Ferrazzi E, Frigerio L, Savasi V, et al. Vaginal delivery in SARS-CoV-2-infected pregnant women in Northern Italy: a retrospective analysis. BJOG, 2020,127(9):11161121

17 Scorzolini L, Corpolongo A, Castilletti C, et al. Comment on the Potential Risks of Sexual and Vertical Transmission of COVID-19. Clin Infect Dis, 2020, 71(16):2298

18 Zhang ZJ, Yu XJ, Fu T, et al. Novel coronavirus infection in newborn babies aged $<28$ days in China. Eur Respir J, 2020;55(6):2000697

19 Hantoushzadeh S, Shamshirsaz AA, Aleyasin A, et al. Maternal death due to COVID-19. Am J Obstet Gynecol, 2020,223(1):109 e101-109 e116

20 Costantine MM, Landon MB, Saade GR. Protection by Exclusion: Another Missed Opportunity to Include Pregnant Women in Research During the Coronavirus Disease 2019 (COVID-19) Pandemic. Obstet Gynecol, 2020,136(1):26-28

21 Wang SS, Zhou X, Lin XG, et al. Experience of Clinical 
Management for Pregnant Women and Newborns with Novel Coronavirus Pneumonia in Tongji Hospital, China. Curr Med Sci, 2020,40(2):285-289

22 Zheng F, Liao C, Fan QH, et al. Clinical Characteristics of Children with Coronavirus Disease 2019 in Hubei, China. Curr Med Sci, 2020,40(2):275-280

(Received Jun. 15, 2020; accepted Mar. 4, 2021) 